\title{
EL HOMBRE DETRÁS DE LA LEY: ACERCA DE LA CARACTERIZACIÓN DE LICURGO EN LA VIDA DE PLUTARCO*
}

\author{
Analía V. Sapere \\ UBA-CONICET \\ analiasapere@gmail.com
}

\section{THE MAN BEHIND THE LAW: THE CHARACTERIZATION OF LYCURGUS IN PLUTARCH'S LIFE}

\begin{abstract}
RESUMEN: Nuestro trabajo se propone dar cuenta de las particularidades de la figura de Licurgo en la Vida de Plutarco, con el fin de demostrar que el biógrafo basa su opinión favorable acerca de éste en la coherencia ética del personaje, pues el éxito de su legislación no está garantizado por la imposición de normas de manera externa, sino en el hecho de que él mismo encarna aquellos valores que pretende inculcar en la población.

PALABRAS CLAVE: Plutarco, Vidas paralelas, Licurgo, Esparta
\end{abstract}

\begin{abstract}
This paper aims to describe the image of Lycurgus in Plutarch's Life. We intend to demonstrate that the biographer bases his positive opinion on the moral coherency of the character, since the success of his legislation is guaranteed not by external coercion but by the fact that he himself embodies the values which he wants the population to apprehend.
\end{abstract}

KEYWORDS: Plutarch, Parallel Lives, Lycurgus, Sparta

RECIBIDO: 24.02.2014. ACEPTADO: 09.06.2014

El legislador Licurgo, retratado por Plutarco en las Vidas Paralelas, es uno de los personajes más destacados de la historia legendaria de Esparta. Su importancia radica en las reformas que se le atribuyen, basadas en las restricciones a la riqueza, la defensa del bien público y el haber modelado una sociedad disciplinada

* Una primera versión de este artículo fue presentada en las V Jornadas sobre el Mundo Clásico, Buenos Aires, Universidad de Morón, 2011. 
militarmente a partir de un estricto sistema educativo, cambios de gran profundidad que han tenido una incidencia innegable en el desarrollo de la sociedad espartana. Nuestro trabajo está orientado a analizar, en primer lugar, de qué modo estas medidas han sido acogidas por los ciudadanos de acuerdo con la visión de Plutarco y, a su vez, de qué forma, para el biógrafo, los ciudadanos han influido en las decisiones de Licurgo, lo que nos permitirá delinear, en definitiva, cómo se configura el vínculo entre ambos. A partir de ello, y sobre la base de un análisis textual, daremos cuenta de las particularidades de la figura del legislador, con el propósito de mostrar que Plutarco basa su opinión favorable acerca de éste en el hecho de haber construido su relación con el pueblo en un tipo de legitimidad emanada de su propia naturaleza y no de factores externos, dado que él mismo encarna aquellos valores que pretende inculcar en la población.

Las transformaciones políticas y sociales llevadas a cabo por Licurgo han sido radicales. Plutarco relata cómo, luego de un período de anomia, desorden y descontento social ${ }^{1}$, el legislador ha sabido poner en práctica un nuevo sistema de leyes para beneficio del pueblo ${ }^{2}$. La constitución diseñada por Licurgo lleva el nombre de Gran Retra ${ }^{3}$, donde se compendian de manera oral las reformas mencionadas (cap. 13) ${ }^{4}$. No es solamente la redistribución de la tierra, la creación de la gerousía, la estricta educación militarizada, la institución de los syssitia, las restricciones en el lujo, los gastos superfluos y los excesos de los ricos, sino el conjunto de todas estas medidas lo que repercute en el pueblo espartano para forjar una imagen positiva de Licurgo. Como indica Plutarco, los ciudadanos habían padecido recientemente un sistema de gobierno injusto $(2)^{5}$, de tal suerte que el

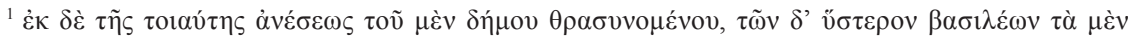

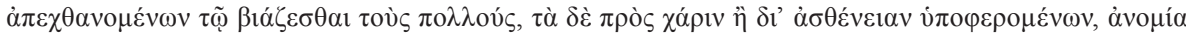

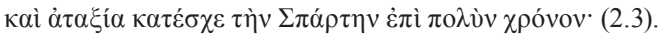

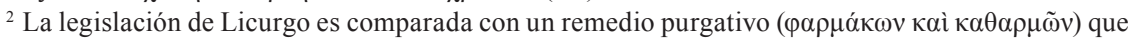

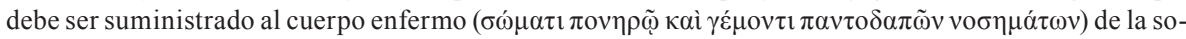
ciedad (5.2). Cf. Koulakiotis (2008: 408) y Ruffy (2011: 153).

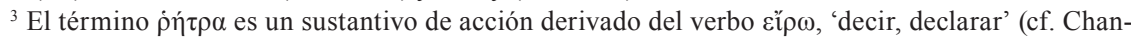

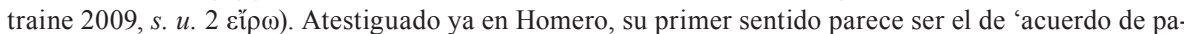
labra'; luego, pasa a designar un 'tratado' o 'compilación de leyes'. Se aplica por antonomasia a las leyes de Licurgo (cf. Liddell-Scott 1996, s. u. ’́ $\tau \rho \alpha$ ). Para un estudio detallado de la Gran Retra en Plutarco, cf. Wade-Gery 1943, 1944a y 1944b. Para un enfoque más general, recomendamos el trabajo de Nafissi 1991. Cf. también Chrimes (1949: 475-89), Hammond (1950), Ogden (1994), Koiv (2000), Maffi (2002), Cartledge (2003: 25 ss.), Raaflaub \& Wallace (2007), Schulz (2011:100-101) y Nafissi (2012a y 2012b) (cf. especialmente el estado de la cuestión sobre el nacimiento del estado espartano). Nafissi 2010 aporta elementos para entender la Gran Retra a través del concepto de la llamada "intentional history", es decir, como un constructo con el propósito de fundamentar la imagen e identidad colectivas. En cuanto al tratamiento del tema en otras fuentes antiguas, cf. Th. 1.18, Hdt. 1.65 y Paus. 3.16.5.

${ }^{4}$ Es importante tener presente el carácter oral de esta legislación, en tanto implica, como destaca Jaeger (1943-1944: 84), menor constricción mecánica y externa de la ley. Cf. a este respecto Nagy (1984: 249-250). Fornis (2009: 3) advierte además que dicha oralidad contribuye a alimentar la imagen mítica de Licurgo.

${ }^{5}$ Plutarco rescata en el capítulo 2 a gobernantes que fueron admirados por los espartanos

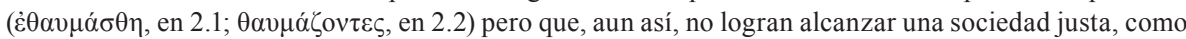


contraste entre ese pasado cercano y el presente y futuro que auguraban las leyes de Licurgo determinaba que estas fueran valoradas aún más ${ }^{6}$. Plutarco subraya especialmente el respeto que el legislador inspiraba en la población $(3.7)^{7} \mathrm{y}$, a su vez, nos enteramos de que Licurgo sentía un profundo cariño hacia su pueblo, pues lo tenía siempre presente en las decisiones que tomaba, dado que su anhelo principal era conseguir la felicidad de la ciudadanía basada en la igualdad y en la unión, por lo que todas sus medidas tendían a ese objetivo. En efecto, Plutarco hace hincapié en esta intención de Licurgo, repitiendo con un vocabulario bastante concreto y enfático el deseo por parte del legislador de que el conjunto de ciuda-

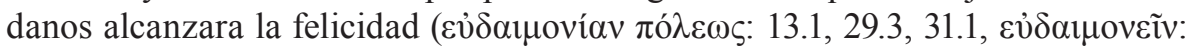

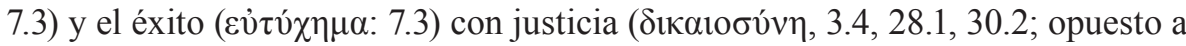

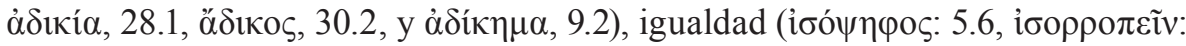

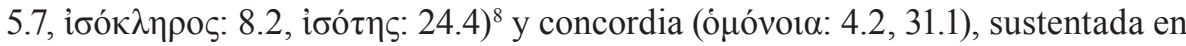

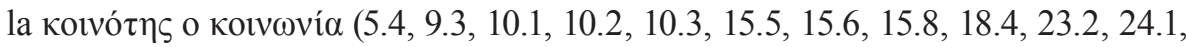
25.39). Se observa asimismo el uso reiterado de vocablos que comienzan con el

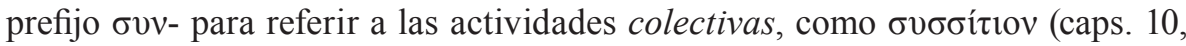

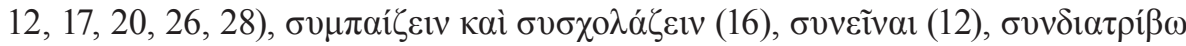

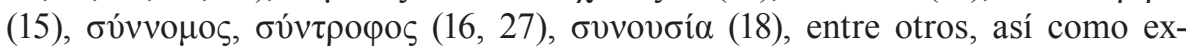
presiones que contienen el pronombre recíproco $\dot{\alpha} \lambda \lambda \hat{\eta} \lambda \omega \mathrm{v}$, para aludir al vínculo entre los ciudadanos, quienes viven en igualdad unos con otros ( $\zeta \tilde{\eta} \nu \mu \varepsilon \tau^{\prime}$

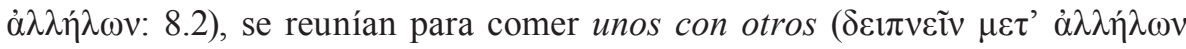

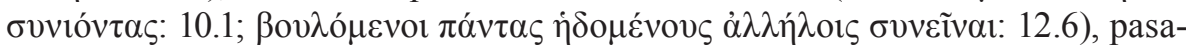

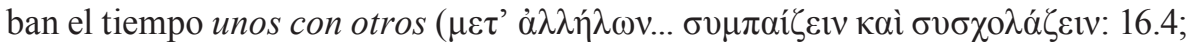

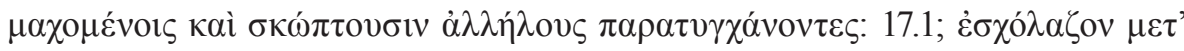

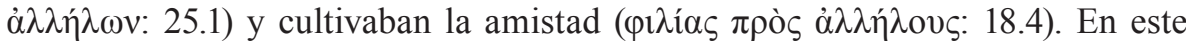
sentido, hay un pasaje más que elocuente en el capítulo 25 , donde queda clara la preeminencia de lo público sobre lo privado:

el legendario rey Soo (cf. también Paus. 3.7.1, Hdt. 7.204; 8.131) y Euripon (cf. también Paus. 3.7.1). Cf. Richer (2001: 16).

${ }^{6}$ Para completar el panorama histórico, Plutarco menciona también a los sucesores de Licurgo, quienes desvirtúan sus políticas (capítulos 6,7 y 30, por ejemplo), con lo que exalta todavía más su figura (idea que el biógrafo mantiene en las vidas de otros espartanos, como Lisandro y Agesilao). Como dice Oliveira Silva (2004: 101): "Para Plutarco, a história de Esparta conheceu dois momentos bastante distintos: a época em que os cidadãos observavam os preceitos licúrgicos e o período de abandono a eses preceitos, iniciado com a Guerra do Peloponeso". Oliveira Silva (2007: 76) también menciona el procedimiento de contextualización que opera en el planteo del biógrafo: "Em Plutarco, o periodo arcaico de Esparta tambén remete à desordem social, então percebemos que o autor beócio traça paralelo entre as histórias dessas cidades e narra como elas encontraram soluções distintas para problemas similares. Com isso, Plutarco nos ensina que as ações devem ser contestualizadas, cada uma delas tem sentidos diferentes, pois estão subordinadas ao seu meio".

${ }^{7}$ Se dice, por ejemplo, que los ciudadanos lo extrañaban cuando se ausentaba por largo tiempo (Plu. Lyc. 5), dando cuenta del componente afectivo en la relación.

${ }^{8}$ Meier (2006) realiza un interesante análisis de la evolución de la idea de igualdad en Esparta, que encuentra sus orígenes en tiempo de Licurgo. Cf. también Daverio Rocchi (2013). 
En definitiva, acostumbró a los ciudadanos a no querer ni saber vivir

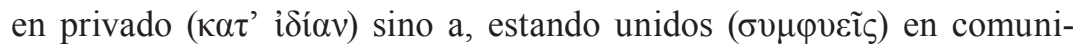

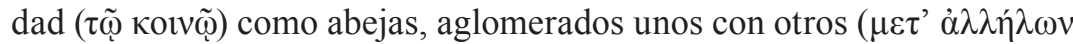

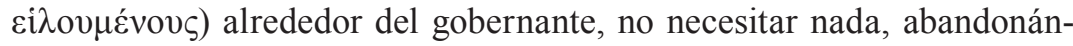
dose a ellos mismos por el entusiasmo y el deseo de honor, y ser por completo de la patria $(\pi \alpha \tau \rho i ́ \delta o \varsigma) .(25.3)^{9}$

La herramienta fundamental para lograr semejante cohesión social es la educación $\left(\pi \alpha 1 \delta \varepsilon i \alpha^{10}\right)$, que Licurgo consideraba "la tarea más grande y bella de un le-

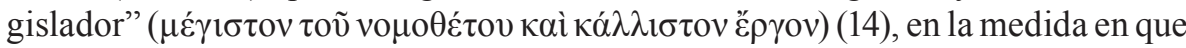

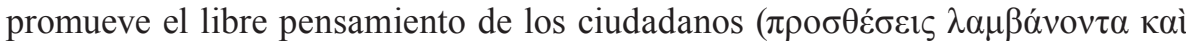

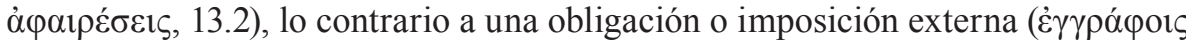

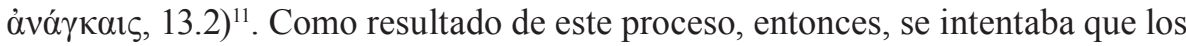
espartanos aprendieran a vivir en una sociedad igualitaria y con estrechos lazos de pertenencia, anteponiendo el bien común al interés particular. Una vez lo-

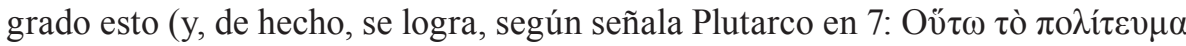

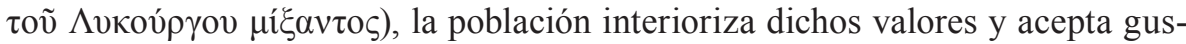
tosa la nueva legislación. Es decir que la fusión entre los ciudadanos se traduce, en última instancia, en la fusión entre los ciudadanos y Licurgo, que es, también, la fusión de toda Esparta.

Desde luego que en dicha representación está presente un fuerte tono de idealización, en consonancia con lo que se conoce como mirage spartiate. La expresión fue acuñada por Ollier (1933), a fin de describir el fenómeno que pretendía favorecer la imagen de los lacedemonios en Grecia antigua, a partir de la distorsión de hechos históricos así como la invención completa de eventos. Se creó, de este modo, una sólida tradición en el mundo griego, con presencia en numerosos

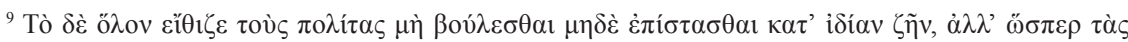

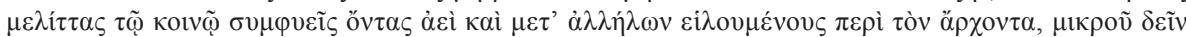

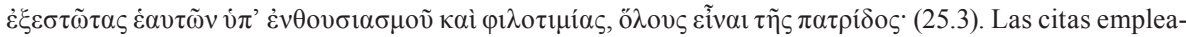
das en el trabajo son de la edición de Flacelière-Chambry-Juneaux (1993). Las traducciones son nuestras.

${ }^{10}$ De la mano de la $\pi \alpha \iota \delta \varepsilon i ́ \alpha$ se desarrolla el sistema de normas, la famosa $\alpha \gamma \omega \gamma \eta ́$ espartana (cf. 5.1; 13.1; 22.1), que trataremos más adelante.

${ }^{11}$ McGlew (1993: 124) se refiere al éxito de esta política de cohesión ciudadana: "A Lycurgus could succeed where Solon failed because Lycurgus existed in the collective imagination of the Spartans, who celebrated the reality of their own political achievement, their collective alienation of personal and immediate control of their affairs, when they remembered Lycurgu's essentially fictional story. In this sense, Delphi was literally correct that Lycurgus was more than a mortal man. If he had been anything less than a god -that it, if he were not the imaginative construction of he entire city- his task would have proved impossible. Any political figure could write his own story, as Solon did more deliberately than most, but none could himself make that story into a collective possession". Haciendo una comparación con Numa, concluye Pérez Jiménez (2012: 8): “mientras Numa escribe sus leyes en piedra y no en el espíritu de los romanos, el programa de Licurgo es esencialmente educativo. El sabio espartano graba las leyes en el alma de los jóvenes, que es la forma más eficaz para salvaguardarlas, puesto que su conocimiento genera la voluntad de cumplirlas; eso las hizo perdurables, y para ello él mismo se sacrificó con el destierro, atando a los espartanos con el juramento". 
testimonios de la antigüedad. A este respecto, Whitby (2002: 11) señala que, después de Ollier, no es posible estudiar la historia de Esparta sin prestar atención a los efectos de distorsión de la tradición idealizadora. Lo curioso es que no se trata solamente de un artilugio propagandístico de parte de los espartanos, sino que el fenómeno se extiende fundamentalmente a testimonios fuera de Lacedemonia. Cartledge (2003: 170) destaca tres elementos centrales de la versión antigua del mirage; en primer lugar, la construcción de un tiempo utópico en el que Esparta estaba desprovista de desórdenes internos y luchas civiles ( $\sigma \tau \alpha ́ \sigma 1 \varsigma)$; luego, el extremo respeto por las leyes de Licurgo (que es, a su vez, el sustento de la situación utópica de paz); en tercer lugar, la idea de que dicha legislación afectaba a cada aspecto de la vida de los ciudadanos, tanto en el ámbito público como en el privado $^{12}$. Los temas de la idealización aparecerán, pues, en la biografía de Plutarco, en la medida en que son de provecho para el tratamiento literario de la figura de Licurgo. Asimismo, encontraremos en la caracterización componentes míticos que pueden rastrearse no sólo en la cultura espartana, sino en muchas otras sociedades antiguas, que adjudican a sus primeros legisladores características heroicas y hasta sobrenaturales ${ }^{13}$; de algún modo, todavía sigue presente el elemento teonómico, en tanto que el respeto a las leyes emana de la veneración a los dioses, que fueron quienes las legaron a los hombres. En el caso de Licurgo, es Apolo, mediante el oráculo de Delfos (5.3) ${ }^{14}$, quien le otorga la facultad de crear las leyes ${ }^{15}$ y de este modo contribuye a legitimar su práctica: Licurgo no es un dios, pero sus vínculos con lo divino lo hacen portador de un status diferenciado respecto de los demás hombres ${ }^{16}$.

Más allá de esta idealización, encontramos también matices: Plutarco no deja de mencionar los rencores que despertaban estas reformas entre los envidiosos,

${ }^{12}$ Cf. Ollier (1933), Tigerstedt (1974), Rawson (1969) y Cartledge (2003: 169-184).

${ }^{13}$ Recordemos que la figura de Licurgo se asocia míticamente con la de Heracles; dice Granitz (2011: 5) al respecto: "Lycurgus and Aeneas/Romulus, themselves anthropomorphic typifications of the character of their founded poleis, identified with Heracles' purpose, bringing justice and civilization, and sought his destiny, apotheosis, through their founding labors". Cf. especialmente el capítulo dedicado a Esparta (37-54). Acerca de la idealización del accionar de Licurgo, cf., por ejemplo, Ollier (1933), Sancho Rocher (1990), Ruzé (2010), y también Jaeger (1943-1944: 84), quien afirma que "la figura del gran estadista y pedagogo Licurgo es una interpretación idealizadora de la vida de Esparta, desde el punto de vista de los ideales educadores de la filosofía posterior". Para un análisis de los tópicos recurrentes en la caracterización de los legisladores arcaicos, cf. Holkeskamp (1992: 52), McGlew (1993: 87-123), Rodrigues (2012: 68 ss.), tópicos que son compartidos con la imagen de los míticos бoøoí griegos (Tell [2011: 85], Wallace [2012]).

${ }^{14}$ El oráculo también es referido por Hdt. 1.65 y Diod. 7.12.1. Cf. Andrewes (1928).

${ }^{15}$ En efecto, lo primero que hace Licurgo, antes de dedicarse a su labor transformadora, es ofrecer sacrificios y consultar a la divinidad (cap. 5); la respuesta es positiva: la Pitia lo llama "amado de los dio-

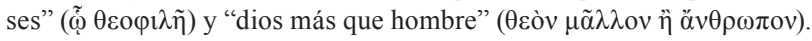

${ }^{16}$ Seung (1996: 8 ss.) explica la diferencia entre un sistema de leyes teonómico y antroponómico; el de Esparta estaría en el medio de ambos. Cf. también Cartledge (2003: 30) y Chirassi Colombo (2008: 282 ss.), quienes analizan el origen divino del poder legislador de Licurgo, pero le adjudican a este y no al dios la invención de las leyes. 
los ambiciosos de poder y los ricos, pues eran quienes más se veían afectados por ellas. Pero las acusaciones hacia Licurgo por parte de estos grupos parecen, desde la mirada del biógrafo, no tener fundamento, sino, más bien, estar basadas en mezquinos intereses. En el comienzo de su carrera pública, por ejemplo, son los familiares más cercanos quienes, ansiosos por llegar al poder, lo calumnian ${ }^{17}$. Las críticas también provienen de los sectores acomodados (oi \&ű $\pi \circ \rho{ }^{18}$ ), con lo que queda claro en la biografía que de ningún modo responden a la opinión popular. En relación con esto, hay un episodio concreto en el que nos detendremos, el incidente con Alcandro, pues resulta ilustrativo del propósito plutarqueo de despejar cualquier duda sobre la integridad de nuestro personaje.

Leemos en el capítulo $11^{19}$ que el odio hacia Licurgo había crecido fuertemente entre los ricos luego del establecimiento de los syssitia ${ }^{20}$. Esto se ve representado en una acción concreta de violencia en contra de Licurgo, quien es atacado en el ágora por un numeroso grupo de ciudadanos acaudalados, enfurecidos por esta medida. Si bien Licurgo escapa con éxito de la multitud y salva su vida al refugiarse en el templo de Atenea Calcieco, un joven llamado Alcandro

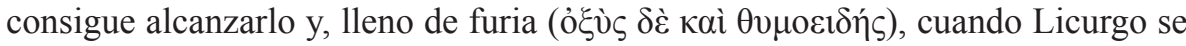
está dando vuelta, lo ataca con su bastón y le quita un ojo. Alcandro es contenido por los presentes (avergonzados además por semejante acto de crueldad) y entre-

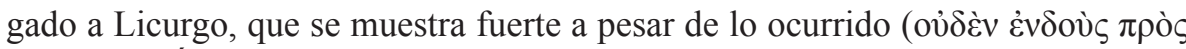

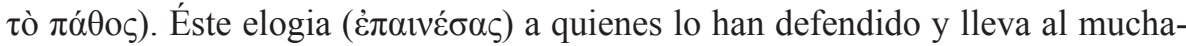
cho a su casa; pero, en lugar de infligirle una pena acorde con el ataque, le hace una propuesta inesperada: trabajar junto a él. Hasta aquí, el episodio exhibe simplemente la grandeza de Licurgo, hombre generoso y pacífico, capaz de perdonar a su enemigo al punto de ofrecerle un lugar a su lado. Asimismo, se ve el cambio

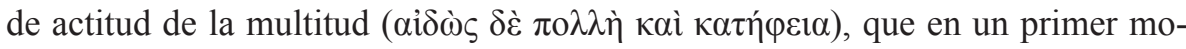

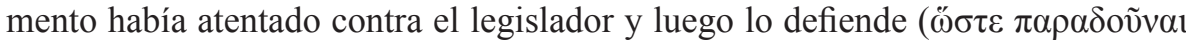

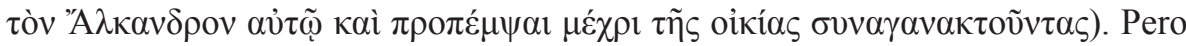
lo más interesante radica en el modo en el que continúa la historia:

Este [Alcandro], que no era de naturaleza vil, hacía lo que se le ordenaba en silencio y, al permanecer al lado de Licurgo y compartir su modo

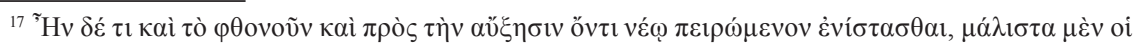

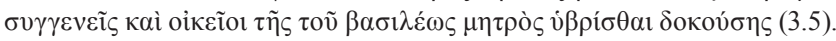

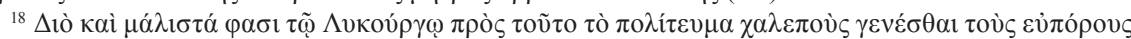

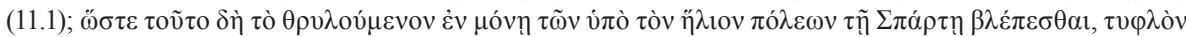

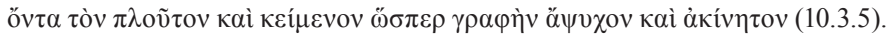

${ }^{19}$ El episodio también es referido por Ael. VH 13.23, Val. Max. 3.2 y Paus. 3.16. Soares (2011: 60) lo toma en su estudio sobre los niños y jóvenes en las Vidas paralelas como ejemplo de uno de los tratamientos "psicológicos" de Plutarco. Cf. también Piccirilli (1981). El episodio es, por cierto, un ejemplo del dominio de los pathêmata en la cultura espartana. Cf. Debnar (2001: 148), Ogden (2008: 247-249) y Richer (2012).

${ }^{20} \mathrm{Se}$ trata de las comidas comunitarias tendentes a igualar a ricos y pobres y a estrechar los lazos entre los ciudadanos. Nos referiremos a ellas más adelante.
} 
de vida, en el conocimiento de su mansedumbre y tranquilidad de espí-

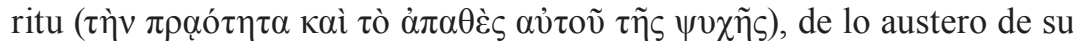

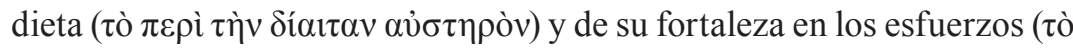

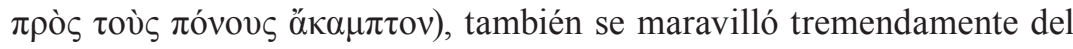
varón, y a los familiares y amigos les decía que Licurgo no era duro ni arrogante, sino que aquel, solamente, era amable y manso con los demás

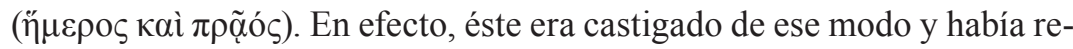
cibido una pena tal que, de joven peligroso y arrogante, se volvió un hom-

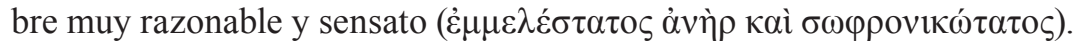
$(11.3 .1-11.4 .4)^{21}$

Entendemos que Plutarco expone los pormenores de este relato, en principio, de poca relevancia histórica, para demostrar cuán infundadas eran las acusaciones de los grupos minoritarios; como consecuencia de su servicio junto a Licurgo, el joven Alcandro no sólo modifica su opinión prejuiciosa, sino que además -y fundamentalmente- comprueba los beneficios de las reformas impulsadas por él, porque conoce ahora (al igual que nosotros, lectores) su fundamento primordial: el propio legislador encarna esos mismos valores en los que se inspiran dichas medidas. Alcandro descubre que Licurgo posee la virtud de la mansedumbre y tranquilidad de espíritu ( $\pi \rho \alpha o ́ \tau \eta \varsigma$ y $\dot{\alpha} \pi \alpha \theta \eta \dot{\varsigma} \varsigma \tau \tilde{\eta} \varsigma \psi v \chi \tilde{\eta} \varsigma)$; es mesu-

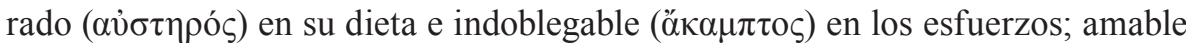

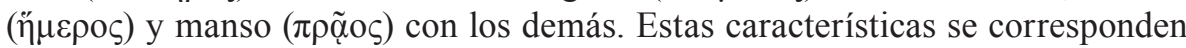
punto por punto con los valores propugnados por Licurgo en la nueva legislación, como procederemos a analizar.

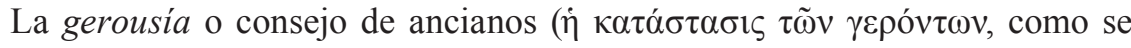
la llama en 5.6.2-3), primera medida dentro de la constitución espartana (5), fue concebida para equilibrar el poder de la monarquía por un lado y los excesos del pueblo por otro: Platón, citado por Plutarco (5.6.3-6), tiene esta opinión (Leyes 691e): “[el consejo de ancianos] mezclado $(\mu \chi \theta \varepsilon \tilde{\varepsilon} \sigma \alpha v)$ con el exaltado gobierno de

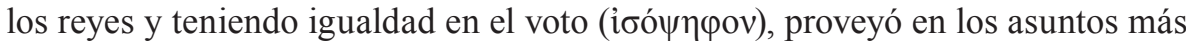

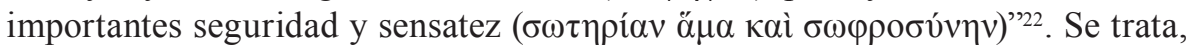
pues, de una institución que aspiraba a encontrar la mesura, característica que,

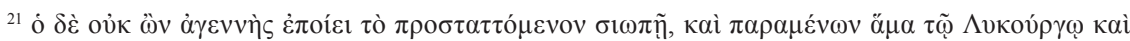

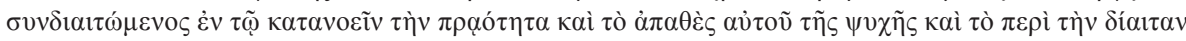

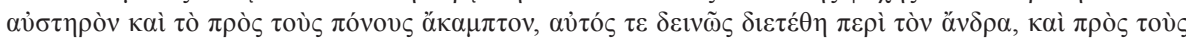

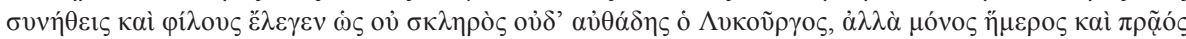

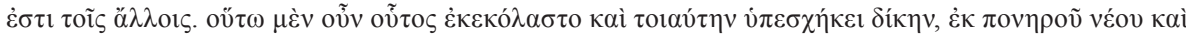
$\alpha$ también en el capítulo 7 de los Ȧ énfasis la furia y violencia del pueblo.

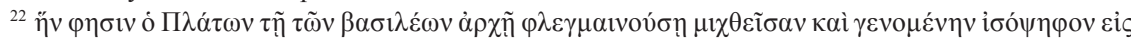

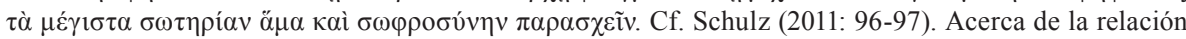
entre las ideas políticas de Platón y el sistema espartano, cf. Levy (2005), Klosko (2006), Liebert (2009), Futter (2012) y González García (2012).
} 
como hemos visto, se atribuye a la personalidad de Licurgo ${ }^{23}$. Este mismo espíritu tienen, a su vez, las leyes vinculadas con la redistribución de la riqueza (ò $\tau \tilde{\eta} \varsigma$ $\gamma \tilde{\eta} \varsigma \dot{\alpha} v \alpha \delta \alpha \sigma \mu$ ós: 8), pues pretenden llegar a una situación de igualdad comunita-

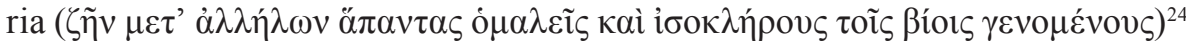

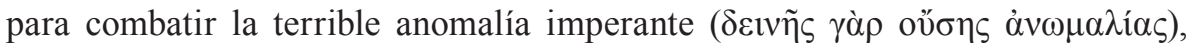

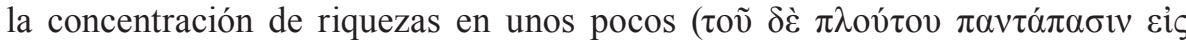

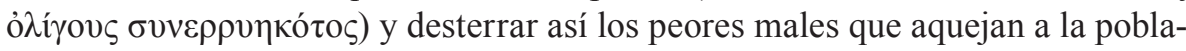

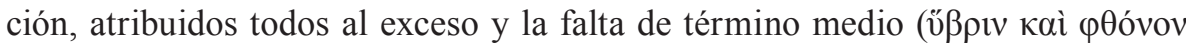

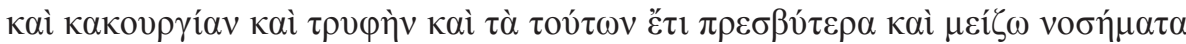

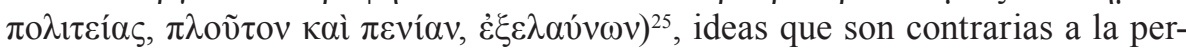

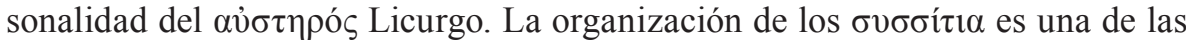
medidas más emblemáticas en este sentido, puesto que establece que todos los ciudadanos deben comer conjuntamente y recibir iguales raciones de alimento

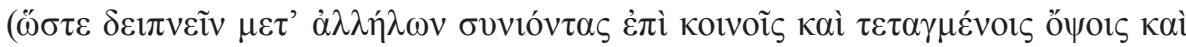
бıtíoıs: 10.1.4-6) ${ }^{26}$. De esta manera, Licurgo buscaba combatir la ostentación y la desmesura de unos pocos adinerados y reducir las diferencias entre los ciudadanos ricos y los pobres $^{27}$. En palabras de Plutarco:

[logró] principalmente que el dinero no fuera codiciado, como dice Teofrasto, y transformarlo en algo sin valor con la comunidad de las co-

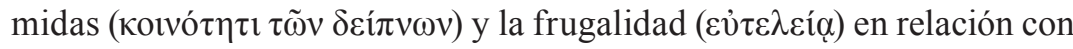
la dieta; pues no era posible ni el disfrute ni la vista o exhibición de un gran preparativo, ya que el rico iba a la misma comida que el pobre $(10.2 .5-10.3 .4 .)^{28}$.

Ya hemos leído en el capítulo 11 que Licurgo destaca por la moderación de su dieta, en lo que parecía un simple dato de color o imagen superflua, pero que

23 "La Gran Retra constituye un instrumento legislativo fundamental que dota al estado espartano de un notable equilibrio interno, apaciguando las tensiones socioeconómicas en el seno de la clase dirigente y la amenaza de la tiranía (régimen político que Esparta nunca conoció)." Cf. Fornis (2009: 4).

${ }^{24} \mathrm{Cf}$. en 8.4.11-12 la metáfora de los hermanos para aludir a la manera igualitaria en la que se ha dis-

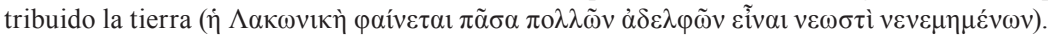

${ }^{25}$ Se reconoce en esta imagen de igualdad una fuerte idealización. Hodkinson (2000) señala que se trata de una tradición inventada, surgida a fines del siglo v por parte de las clases altas atenienses desencantadas con su propio contexto democrático, propiciada, además, por un ambiente filosófico en el que se buscaba encontrar la naturaleza del "estado ideal". Cf. también Hodkinson (2007).

${ }^{26}$ Cf. X. Lac. 2.5; 5.1-9; 6.4; Arist. Pol. 1271a 27-37, 1272a 12-21, Plb. 6.48.3. Plácido (2011) refiere la importancia de las comidas comunitarias en la constitución de las ciudades griegas como recurso de cohesión previa al establecimiento oficial del culto. Cf. también Oliva (1983: 31 ss.), Figueira (1984), Lavrencic (1993), Casillas y Fornis (1994), Link (1998), Nafissi (2000), Powell (2014: 26-50).

${ }^{27}$ Cf. Rabinowitz (2009) y Ruffy (2011).

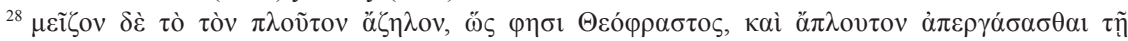

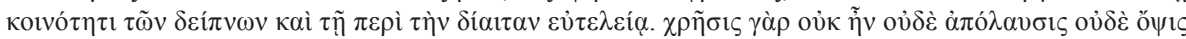

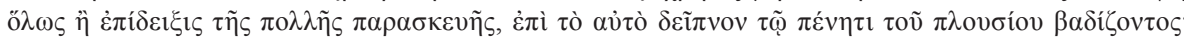
(10.2.5-10.3.4). 
a la luz de esta referencia cobra otro sentido, dado que observamos una consonancia entre la práctica privada del legislador y el fundamento de la institución de los $\sigma v \sigma \sigma i ́ \tau 1 \alpha$.

Otro rasgo importante de la personalidad de Licurgo es su fortaleza de espíritu, pues se dice que es ö $\alpha \alpha \mu \pi \tau$ co los esfuerzos; esto queda reflejado en el tipo

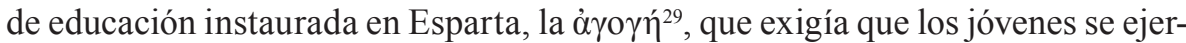
citaran con dureza ${ }^{30}$. En 14.2, Plutarco afirma que Licurgo deseaba eliminar toda

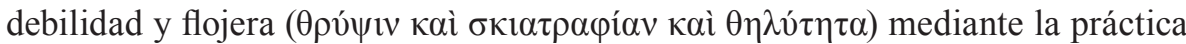
de actividades gimnásticas y deportivas, incluso por parte de las mujeres, a quienes también se exponía a fatigas físicas ( $\tau$ à $\mu \varepsilon ́ v \gamma \varepsilon \sigma \omega ́ \mu \alpha \tau \alpha \tau \tilde{\omega} v \pi \alpha \rho \theta \varepsilon ́ v \omega v \delta \rho o ́ \mu o r \zeta$

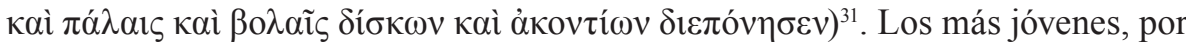
supuesto, eran la base de esta estricta educación militar (16-17), que eran criados desde muy pequeños para ser temerarios ( $\alpha \theta \alpha \mu \beta \eta ́ \varsigma, ~ \ddot{\alpha} \varphi о \beta o \varsigma)$, de buena contex-

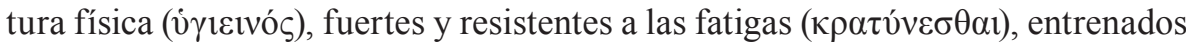

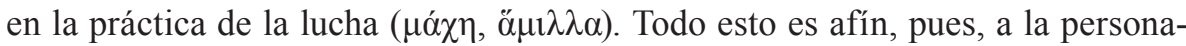
lidad aguerrida de Licurgo ( $\pi \circ \lambda \varepsilon \mu 1 \kappa \omega ́ \tau \alpha \tau o v)$ a la que también alude Plutarco en 23.1, tomando a Hipias como fuente, así como a su experiencia en asuntos bélicos, constatada por Filostéfano ${ }^{32}$.

En esta agogé no solamente se propician valores guerreros, como es esperable, sino también intelectuales; tal es el caso de la instrucción que se impartía a los jóvenes en materia de retórica. Leemos en 19.1:

Enseñaban a los niños a valerse en el discurso de agudeza mezclada

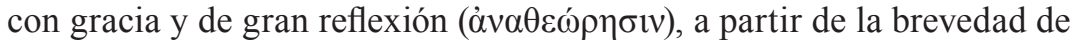

\footnotetext{
${ }^{29}$ Plutarco es, de hecho, la fuente más completa acerca de ella. Es importante destacar, sin embargo, que la crítica más actual considera que la agogé tal como se la conoce tradicionalmente es una creación posterior, de época helenística y romana. Cf. Kenell (1995: 23 ss.; 98-114), Hodkinson (2005: 51), Ducat (2006), y Soares (2011: 66 ss.).

${ }^{30}$ Más allá del aspecto vinculado con la educación militar, Licurgo también es esforzado en lo que hace a su labor por el bien popular. En el capítulo 4, por ejemplo, se relatan los viajes que realizó con el fin de conocer las costumbres de diferentes pueblos e indagar acerca de las formas de gobierno y sistemas legislativos, para tomar ejemplo de ellos en vistas a confeccionar la nueva constitución espartana, tarea por demás ardua. Cf. Schulz (2011: 251-252).

${ }^{31}$ Acerca de estas labores femeninas, cf. Pomeroy (2002: cap. 1), Oliveira Silva (2005) y Soares (2011: 71-2). Cf. además Redfield (1977), Cartledge (1981), Mossé (1983: 80-89), Bradford (1983), Dettenhofer (1993), Zweig (1993), Blundell (1995: 150-159), Fantham et alii (1995) y Millender (1999). Plutarco

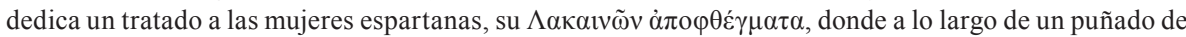
anécdotas destaca su obediencia, apego a la ley, valor, temple y la estima hacia la virtud bélica, en consonancia con lo expresado en esta biografía. Sobre las implicancias sociales y económicas de este fenómeno, cf. Fleck y Hanssen 2009.

${ }^{32}$ También hay otra versión (de Demetrio Falereo), aunque relegada a un segundo plano en el relato, según la cual Licurgo no emprendió ninguna acción bélica, sino que mantuvo la paz durante la institución de la Gran Retra (23.2). Plutarco no parece estar de acuerdo con ella (motivo por el cual la menciona en segundo término), pero su rigor de investigador lo obliga a cumplir con sus lectores y ofrecerles todo el material a disposición.
} 


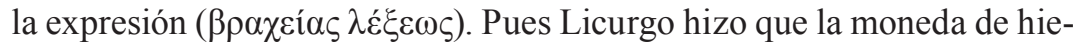
rro, según se dice, tuviera poco valor respecto de su mucho peso ${ }^{33}$; en cambio, la moneda de la palabra, a partir de una expresión simple y corta

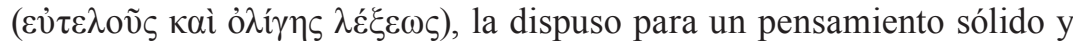

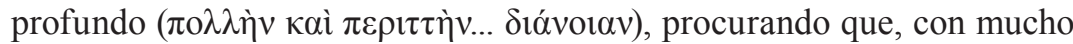

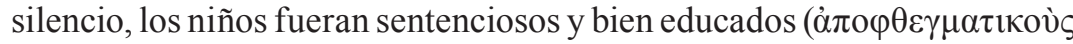

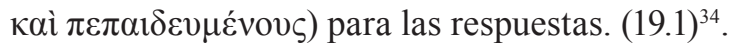

Unas líneas más abajo (19.3), Plutarco afirma que Licurgo poseía también la

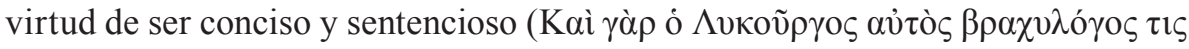

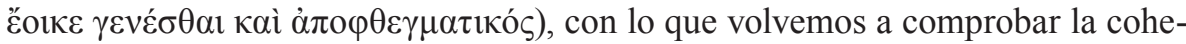
rencia entre sus decisiones legislativas y su naturaleza ${ }^{35}$. De la misma manera, se ofrecían a los jóvenes enseñanzas musicales y poéticas (21), también del agrado de Licurgo, según leemos en el capítulo $4^{36}$.

Hasta aquí hemos visto que Plutarco se vale del episodio de Alcandro para oponerse a las críticas hacia Licurgo. De este modo, minimiza las opiniones contrarias a él expuestas a lo largo de la biografía y potencia su imagen positiva, pues identifica las características de la personalidad de Licurgo con las exitosas reformas que terminaron favoreciendo a la población espartana. Como afirma Boulet (2005: 253), "Sparta is made to reflect Lycurgus' soul". La coherencia avala las medidas de Licurgo, pensadas para el pueblo a partir de una convicción genuina y no a partir del oportunismo político o de la demagogia, lo que practican, muestra Plutarco, otros líderes espartanos. De Euriponte, por ejemplo, dice que (2.2) "fue el primero que dejó de lado en la corona lo excesivamente monárquico, ganando el favor popular $(\delta \eta \mu \alpha \gamma \omega \gamma \tilde{\omega} v)$ y congraciándose con la multitud ( $\chi \alpha \rho ı \zeta o ́ \mu \varepsilon v o \varsigma$

${ }^{33}$ Como señala Plutarco en el capítulo 9, Licurgo había impulsado una reforma monetaria que consistía en sustituir el uso de las monedas de oro por monedas de hierro de baja calidad (dado que se templaban con vinagre), mucho más pesadas e imprácticas; de este modo, pretendía disminuir la codicia de los ciudadanos y los gastos superfluos, dado que nadie estaba interesado en tenerlas o recibirlas (mucho menos robarlas), pues eran de difícil transporte y almacenamiento y de poco valor por fuera de Esparta. Es interesante contrastar la situación de Esparta en este período con la que se ilustra en el capítulo 17 de la Vida de Lisandro. Cf. Mossé 1999.

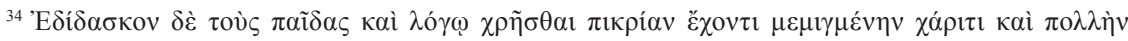

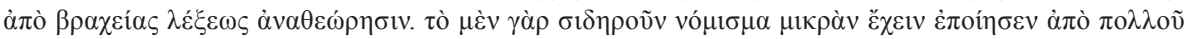

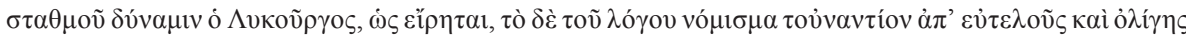

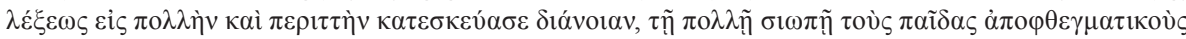

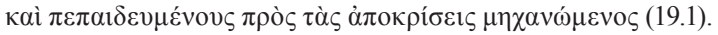

${ }^{35}$ Acerca del laconismo espartano, cf. Schmitz (2006) y Fornis (2012). Este último rescata el valor sapiencial y pragmático de este tipo de expresión breve y concisa, contra las interpretaciones estereotipadas y prejuiciosas que hablan de un casi analfabetismo de los espartanos frente al florido estilo de la retórica ateniense. Plutarco también se refiere a ello en De garrulitate 17 y 21.

${ }^{36}$ Nos referimos a su preferencia por los poemas de Homero, en los que encontraba la combinación perfecta entre arte, educación y política. Esto nos habla, a la vez, del posicionamiento de Esparta como sede de cultura para la época. Cf. Ferrari (2008: 9). Mosconi (2009) traza un interesante paralelo entre la armonía musical y la armonía social y política en Plutarco, que puede aplicarse perfectamente a la biografía de Licurgo. 


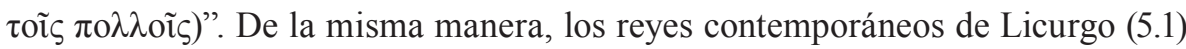
estaban deseosos de obtener el favor popular con la única intención de que la

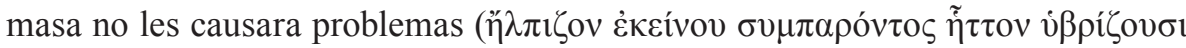

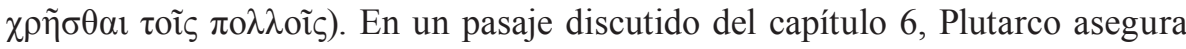
que Polidoro y Teopompo (reyes del siglo VII ${ }^{37}$ ), limitaron la influencia del pueblo en las deliberaciones de la asamblea y, con una injusta manipulación, convencían a la ciudadanía de que lo decidido había sido dictaminado por los dioses (6.4-6.5). La constitución de Licurgo también tenía su principal fundamento en la voluntad divina, pues se origina a partir del oráculo de Delfos, como ya hemos apuntado, lo que le otorga, desde luego, legitimidad; pero la legitimidad no surge solamente de esto, sino de una personalidad basada en los mismos valores defendidos públicamente, valores que se consolidan, como hemos visto, con una educación que fomentaba el espíritu ciudadano ${ }^{38}$. En el caso de Polidoro y Teopompo, entonces, se trata, según Plutarco, de una evidente estratagema para engañar a los ciudadanos y mantenerlos así satisfechos.

Se añade además un mérito a la imagen que tiene el pueblo espartano de Licurgo: la dificultad de la implementación de las reformas. No es nada sencillo conseguir la adhesión masiva ante cambios tan drásticos y medidas tan estrictas a nivel político y social. Plutarco reflexiona acerca de ello a la luz de la comparación con Numa, quien atraviesa en Roma una situación muy distinta:

Ciertamente, puesto que, como en la armonía de una lira, uno [Licurgo] tensó la Esparta relajada y licenciosa y el otro [Numa] soltó la vehemencia e intensidad de Roma, la dificultad ( $\chi \alpha \lambda \varepsilon \pi$ ó $\rceil \zeta)$ de la tarea se le presenta a Licurgo; pues no persuadió a los ciudadanos para que se quitasen las corazas y depusieran las espadas, sino para que abandonaran el oro y la plata y se deshicieran de costosas mantas y mesas; y no los persuadió para que, tras cesar la guerra, hicieran celebraciones y ofrendas, sino para que, tras dejar las comidas y bebidas, se esforzaran y ejercitaran en las armas y en las palestras. De donde, uno [Numa] hizo todas las cosas persuadiendo por la indulgencia y honor de los que gozaba;

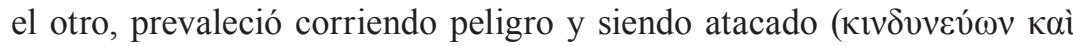

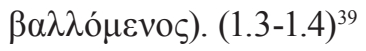

${ }^{37}$ Se discute si Plutarco está aquí manipulando información para desprestigiar a Polidoro y Teopompo, pues esta medida que les atribuye no parece concordar con el espíritu democrático que los caracterizaba.

${ }^{38}$ En Lisandro 17 hallamos un ejemplo de lo contrario, es decir, de un tipo de legislación que apela sólo a la coacción externa, basada en el miedo y no en la aceptación por parte de los ciudadanos. De hecho, se menciona, a modo de contraste, la figura de Licurgo.

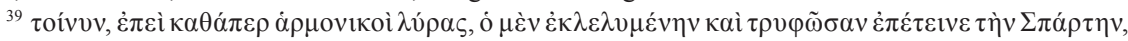

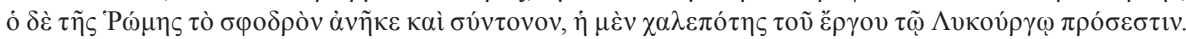

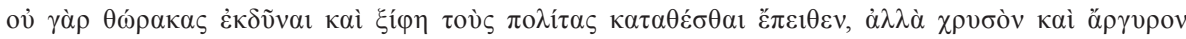

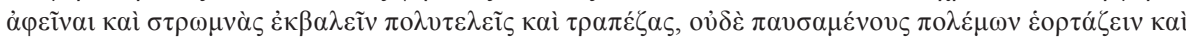


El tipo de medidas adoptadas por Licurgo, entonces, hace más admirable que éste haya conseguido la aceptación popular. Plutarco corrobora este acto de valentía cuando afirma que, para Licurgo (5.3), no sirven las leyes parciales ( $\dot{\varsigma} \tau \tilde{\omega} v$

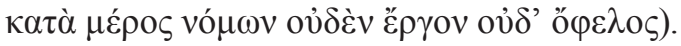

En conclusión, Licurgo trabaja en pro de mejorar la vida de sus ciudadanos y, por su parte, el pueblo responde con beneplácito a las medidas transformadoras del legislador. Se forja, por ende, un vínculo entre el grupo de ciudadanos y el creador de sus leyes. La legitimidad de esta concordia descansa, primero, en el origen divino de las normas y luego, en el sistema disciplinar implantado en todos los niveles de la sociedad. Sin embargo, ninguna de estas reformas hubiera sido exitosa si no las hubiera impulsado Licurgo. Desde el punto de vista de la estructura narrativa, resulta clave a este respecto el episodio de Alcandro, dado que, al hacer un acercamiento al ámbito privado del personaje, nos permite comprobar el interés genuino del legislador por las medidas adoptadas y comprendemos así que estas armonizan a la perfección con sus características personales. Sobre esta base, la nueva legislación tiene solidez, pues es sólido su creador. La cohesión ciudadana que anhela (y logra) Licurgo se refleja, según vimos, en la convicción interna del legislador.

Una última reflexión: si tenemos en cuenta los esfuerzos de Licurgo para priorizar lo comunitario por encima de lo privado, resulta paradójico que sea el individuo quien sustente los logros colectivos. Y es aquí donde debemos recordar la mirada particular de Plutarco respecto del asunto; es el biógrafo el que necesita fundar su paideia en el personalismo, dado que el propósito edificante de las vidas surge, en efecto, de la exhibición de modelos de conducta, con sus virtudes y defectos ${ }^{40}$. En este planteamiento, entonces, es lógico que el legislador sea erigido como ejemplo individual para la conducta colectiva de los espartanos, aunque no parezca ser ese el espíritu de su legislación, más abocada, como vimos, a la valoración de lo comunitario. La imagen de Licurgo que nos presenta Plutarco puede sustentarse en una profunda investigación de su parte, en el cotejo de fuentes y documentos, y en un tratamiento serio de todo ese material; sin embargo, siempre acecha la perspectiva del biógrafo y su subjetividad, que deberá ser incorporada al análisis de los hechos y personajes del mundo antiguo, si queremos enriquecer nuestra lectura crítica.

\footnotetext{
$\theta$

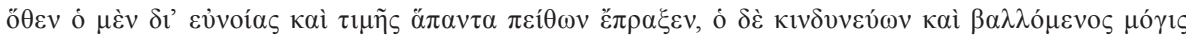

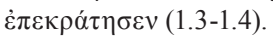

${ }^{40}$ Tema ampliamente tratado en la bibliografía. Cf. a modo de ejemplo Russell (1966: 141), Bosworth (1992: 65) y Pérez Jiménez (2002: 106).
} 


\section{BIBLIOGRAFÍA}

Andrewes 1928: A. Andrewes, "Eunomia”, CQ 32, 2 (1928) 89-102.

Beekes 2010: R. Beekes, Etymological Dictionary of Greek (Boston 2010).

Bitros-Karayiannis 2007: G. Bitros, A. Karayiannis, "Morality, Institutions and Economic Growth: Lessons from ancient Greece", MPRA, Paper $\mathrm{n}^{\circ} 994$ (2007).

Blundell 1995: S. Blundell, Women in Ancient Greece (Cambridge 1995).

Bosworth 1992: A. B. Bosworth, "History and Artifice in Plutarch's Eumenes", en P. A. Stadter (ed.), Plutarch and the Historical Tradition (Routledge 1992) 56-89.

Boulet 2005: B. Boulet, "Is Numa the Genuine Philosopher King?", en L. Blois et al. (eds.), The Statesman in Plutarch's Works, Volume I and II: Proceedings of the Sixth International Conference of the International Plutarch Society, Nijmegen / Castle Hernen, May 1-5, 2002 (Boston 2005) 245-256.

Bradford 1986: A. S. Bradford, "Gynaikokratoumenoi: Did Spartan Women Rule Spartan Men?", The Ancient World 14 (1986) 13-18.

Casillas y Fornis 1994: J. M. Casillas, C. Fornis, "La comida en común espartana como mecanismo de diferenciación e integración social”, ETF (Historia Antigua) 7 (1994) 65-83.

Cartledge 1981: P. A. Cartledge, "Spartan Wives: Liberation or Licence?", CQ 31 (1981) 84-105.

Cartledge 2003: P. Cartledge, Spartan Reflections (California 2003).

Chantraine 2009: P. Chantraine, Dictionnaire étymologique de la langue grecque (Paris 2009).

Chirassi Colombo 2008: I. Chirassi Colombo, "Mythos / Nomos (Il Racconto e la Legge)", en P. Pisi, B. Scarcia (eds.), Religione e Politica: mito autorità diritto (Roma 2008) 278-304.

Chrimes 1949: K. M. T. Chrimes, Ancient Sparta: A Re-examination of the Evidence (Manchester 1949).

Daverio Rocchi 2013: G. Daverio Rocchi, "Virtù spartane: andreia kai homonoia", en La cultura a Sparta in età classica, Atti del seminario di Studi Università Statale di Milano (Trento 2013) 13-26.

Debnar 2001: P. Debnar, Speaking the Same Language: Speech and Audience in Thucydides' Spartan Debates (Michigan 2001).

Dettenhofer 1993: M. Dettenhofer, "Die Frauen von Sparta. Gesellschaftliche Position und politische Relevanz”, Klio 75 (1993) 61-75.

Duff 1999: T. Duff, Plutarch's lives: Exploring Virtue and Vice (Oxford 1999).

Ducat 2006: J. Ducat, Spartan Education. Youth and Society in the Classical Period (Swansea 2006).

Fantham et alii 1995: E. Fantham et alii, "Spartan Women: Women in a Warrior Society", en Women in the Classical World. Image and Text (Oxford 1995) 56-67. 
Feinberg 1970: B. S. Feinberg, "Creativity and the Political Community: The Role of the Law-Giver in the Thought of Plato, Machiavelli and Rousseau", The Western Political Quarterly 23.3 (1970) 471-484.

Ferrari 2008: G. Ferrari (2008), Alcman and the Cosmos of Sparta (Chicago 2008). Figueira 1984: T. J. Figueira, "Mess Contribution and Subsistance at Sparta", TAPhA 114 (1984) 87-109.

Flacelière-Chambry-Juneaux 1993: R. Flacelière, E. Chambry, M. Juneaux (eds.) Plutarque, Vies (t. 1), troisième tirage revu et corrigé par J. Irigoin (Paris 1993).

Fleck-Hanssen 2009: R. K. Fleck, F. A. Hanssen, “'Rulers ruled by women': an economic analysis of the rise and fall of women's rights in ancient Sparta", Economics of Governance 10.3 (2009) 221-245.

Fornis 2009: C. Fornis, "Esparta, ciudad de la virtud y de la guerra", en Historia de tres ciudades: Atenas, Esparta y Utopia, XVI Jornadas Antiqua organizadas por el Centro Koldo Mitxelena de San Sebastián (San Sebastián 2009) 1-13.

Fornis 2012: C. Fornis, "Laconismo frente a retórica. Aforismo y brevilocuencia en el lenguaje espartano", en L. Sancho Rocher et al., Lógos y arkhé. Discurso político y autoridad en la Grecia antigua (Buenos Aires 2012) 49-67.

Futter 2012: D. Futter, "Plutarch, Plato and Sparta", Akroterion 57 (2012) 35-51.

González García 2012: A. González García (2012), "La paideia y la construcción de la República platónica", Historia Autónoma 1 (2012) 21-36.

Granitz 2011: N. Granitz, Heracles and the Foundings of Sparta and Rome (Ohio 2011).

Hammond 1950: N. G. Hammond, "The Lycurgean Reform at Sparta", JHS 70 (1950) 42-64.

Hodkinson 2000: S. Hodkinson, Property and Wealth in Classical Sparta (London 2000).

Hodkinson 2005: S. Hodkinson, "The development of Spartan Society and Institutions in the Archaic Period", en L. Mitchell, P. J. Rhodes (eds.), The Development of the Polis in Archaic Greece (London-New York 2004) 44-54.

Hodkinson 2007: S. Hodkinson, "Five words that shook the world: Plutarch, Lykourgos 16 and appropiations of Spartan communal property ownership in eighteenth-century France”, en N. Birgalias, K. Buraselis, P. Cartledge (eds.), The Contribution of Ancient Sparta to Political Thought and Practice (Athens 2007) 417-430.

Holkeskamp 1992: K. J. Holkeskamp, "Arbitrators, Lawgivers and the 'Codification of Law' in Archaic Greece [Problems and Perspectives]", Mètis. Anthropologie des mondes grecs anciens 7.1-2 (1992) 49-81.

Jaeger 1943-1944: W. Jaeger, "La educación del estado en Esparta", en Paideia: los ideales de la cultura griega (México 1943-1944) 84-102.

Kennell 1995: N. M. Kennell, The Gymnasium of Virtue: Education \& Culture in Ancient Sparta (London - Chapel Hill 1995)

Klosko 2006: G. Klosko, The Development of Plato's Political Theory (Oxford 2006). 
Koiv 2000: M. Koiv, “The Origins, Development and Reliability of the Ancient Tradition about the Formation of Spartan Constitution", Studia Humaniora Tartuensia 1-3 (2000) 1-27.

Koulakiotis 2008: E. Koulakiotis, "Greek Lawgivers in Plutarch: A comparison Between the Biographical Lycurgus and the Rhetorical Alexandermore", en A. Nikolaidis (ed.), The Unity of Plutarch's Work: 'Moralia' Themes in the 'Lives', Features of the 'Lives' in the 'Moralia' (Berlin - New York 2008) 403-422.

Lavrencic 1993: M. Lavrencic, Spartanische Küche. Das Gemeinshaftsmahl der Männer in Sparta (Wien 1993).

Liddell-Scott 1996: H. G. Liddell, R. Scott (1996), A Greek-English Lexicon (Oxford 1996).

Link 1998: S. Link, "Durch diese Tür geht kein Wort hinaus! (Plut. Lyk. 12.8): Bürgergemeinschaft und Syssitien in Sparta", Laverna 9 (1998) 82-112.

Levy 2005: E. Levy, “La Sparte de Platon”, Ktema 30 (2005) 217-236.

Liebert 2009: H. Liebert, "Plutarch's Critique of Plato's Best Regime”, HPTh 30.2 (2009) 251-271.

Maffi 2002: A. Maffi, “Studi recenti sulla Grande Rhetra”, Dike 5 (2002) 195-236.

McGlew 1993: J. F. McGlew, Tyranny and Political Culture in Ancient Greece (Ithaca 1993).

Meier 2006: M. Meier, "Wann entstand das Homoios-Ideal in Sparta?”, en A. Luther et al., Das frühe Sparta (Leipzig 2006) 113-124.

Millender 1999: E. Millender, "Athenian Ideology and the Empowered Spartan Woman”, en S. Hodkinson, A. Powell (eds.), Sparta: New Perspectives (London - Swansea 1999) 355-91.

Mosconi 2009: G. Mosconi, “'Governare in armonia': struttura e significato ideologico di un campo metaforico in Plutarco", en D. Castaldo et al. (eds.), Il sapere musicale e i suoi contesti da Teofrasto a Claudio Tolomeo (Ravenna 2009) 105-128.

Mossé 1983: C. Mossé, La femme dans la Grèce antique (Paris 1983) 80-89.

Mossé 1999: C. Mossé, "Plutarque et le déclin de Sparte dans les 'Vies' de Lysandre et d'Agesilas", Eirene 35 (1999) 41-46.

Nafissi 1991: M. Nafissi, La nascita del Kosmos. Studi sulla storia e società di Sparta (Napoli 1991).

Nafissi 200: M. Nafissi, "Los syssítia espartanos", en A. Pérez Jiménez (ed.), Dieta mediterránea. Comidas y hábitos alimentarios desde la Antigüedad al Renacimiento (Madrid 2000) 21-42.

Nafissi 2010: M. Nafissi, "The Great Rhetra (Plu. Lyc. 6): A Retrospective and Intentional Construction?", en L. Foxhall, H. J. Gehrke, N. Luraghi (eds.), Intentionale Geschichte. Spinning Time in Ancient Greece (Stuttgart 2010) 89-119.

Nafissi 2012a: M. Nafissi, "The foundation of Heraclid Laconia: identities and forms of dependence", en K. A. Raaflaub, H. van Wees, A Companion to Archaic Greece (New York 2012). 
Nafissi 2012b: M. Nafissi, "Esparta”, en C. Fornis (ed.), Mito y arqueología en el nacimiento de ciudades legendarias de la Antigüedad (Sevilla 2012) 25-57.

Nagy 1984: G. Nagy, “Théognis et Mégare. Le poète dans l'âge de fer”, RHR 201.3 (1984) 239-279.

Ogden 1994: D. Ogden, "Crooked Speech: The Genesis of the Spartan Rhetra", JHS 114 (1994) 85-102.

Ogden 2008: D. Ogden, A Companion to Greek Religion (New York 2008).

Oliva 1983: P. Oliva, Esparta y sus problemas sociales (Madrid 1983).

Oliveira Silva 2004: M. A. Oliveira Silva, "Plutarco e a biografia de Esparta", Politeia: Hist. e Soc., Vitória da Conquista 4.1 (2004) 83-103.

Oliveira Silva 2005: M. A. Oliveira Silva, "Plutarco e a participação feminina em Esparta”, Sceculum . Revista de história 12 (2005) 11-20.

Oliveira Silva 2007: M. A. Oliveira Silva, Plutarco e Roma: o mundo grego no Império (Sâo Paulo 2007).

Ollier 1933: F. Ollier, Le mirage spartiate. Étude sur l'idéalisation de Sparte dans l'antiquité grecque de l'origine jusqu'aux cyniques (Paris 1933).

Pérez Jiménez 2002: A. Pérez Jiménez, "Exemplum: the Paradigmatic Education of the Ruler in the Lives of Plutarch", en P. Stadter y L. Van der Stockt (eds.), Sage and emperor: Plutarch, Greek intellectuals, and Roman power in the time of Trajan (98-117 A. D.) (Leuven 2002) 105-11.

Pérez Jiménez 2012: A. Pérez Jiménez, “Nomos y dikaiosyne como criterio de valoración ética en las Vidas Paralelas", en J. Ribeiro Ferreira et al. (eds.), Nomos, Kosmos \& Dike in Plutarch (Coimbra 2012) 5-22.

Piccirilli 1981: L. Piccirilli, "Licurgo e Alcandro: monoftalmia e origine dell' 'Agoge' spartana", Historia 30.1 (1981) 1-10.

Plácido 2011: D. Plácido, "La comensalidad en el origen de las comunidades cívicas griegas", ARYS 9 (2011) 33-47.

Pomeroy 2002: S. Pomeroy, Spartan Women (Oxford 2002).

Raaflaub \& Wallace 2007: K. A. Raaflaub, R. W. Wallace, “'People's Power' and Egalitarian Trends in Archaic Greece", en K. A. Raaflaub et al. (eds.), Origins of Democracy in Ancient Greece (Berkeley - Los Angeles - London 2007) 22-48.

Rabinowitz 2009: A. Rabinowitz, "Drinking from the Same Cup: Sparta and Late Archaic Commensality", en S. Hodkinson (ed.), Sparta: Comparative Approaches (Swansea 2009) 113-91.

Rawson 1969: E. Rawson, The Spartan Tradition in Western Thought (Oxford 1969).

Redfield 1978: J. Redfield, "The Women of Sparta”, CJ 73.2 (1978) 146-161.

Richer 2001: N. Richer, “'Eunomia' et 'eudamonia' à Sparte”, Dike 4 (2001)13-38.

Richer 2012: N. Richer, La religion des Spartiates. Croyances et cultes dans l'Antiquité (Paris 2012).

Rodrigues 2012: A. R. Rodrigues, "Political reforms in the Lives of Lycurgus and Numa: divine revelation or Political lie?", en J. Ribeiro Ferreira et al. (eds.), Nomos, Kosmos \& Dike in Plutarch (Coimbra 2012) 67-83. 
Ruffy 2011: M. V. Ruffy, "Symposium, Physical and Social Health", en F. Klotz, The Philosopher's Banquet: Plutarch's Table Talk in the Intellectual Culture of the Roman Empire (Oxford 2011) 131-157.

Russell 1966: D. A. Russell, “On Reading Plutarch's 'Lives”, $G \& R 13.2$ (1966) 139-154.

Ruzé 2010: F. Ruzé, "L’Utopie spartiate”, Kentron 26 (2010) 17-48.

Sancho Rocher 1990: L. Sancho Rocher, "Homoiótes; los hómoioi de Esparta", Gerión 8 (1990) 45-71.

Schmitz 2006: W. Schmitz, "Die Macht über dis Sprache. Kommunikation, Politik und soziale Ordnung in Sparta", en A. Luther et al., Das frühe Sparta (Leipzig 2006) 89-112.

Schulz 2011: F. Schulz, Die homerischen Räte und die spartanische Gerusie (Düsseldorf 2011).

Seung 1996: T. K. Seung, Plato Rediscovered: Human Value and Social Order (Lanham 1996).

Soares 2011: C. Soares, Crianças e jovens nas Vidas de Plutarco (Coimbra 2011). Stadter 1992: P. A. Stadter (ed.), Plutarch and the Historical Tradition (London 1992).

Tell 2011: H. Tell, Plato's Counterfeit Sophists (Cambridge 2011).

Tigerstedt 1974: E. N. Tigerstedt, The Legend of Sparta in Classical Antiquity (Stockholm-Göteborg-Uppsala 1974) II, 226-264.

Wade-Gery 1943: H. T. Wade-Gery "The Spartan Rhetra in Plutarch Lycurgus VI: A. Plutarch's Text", CQ 37.1/2 (1943) 62-72.

Wade-Gery 1944a: H. T. Wade-Gery, "The Spartan Rhetra in Plutarch Lycurgus VI: B. The Eynomia of Tyrtaios", CQ 38.1/2 (1944) 1-9.

Wade-Gery 1944b: H. T. Wade-Gery, "The Spartan Rhetra in Plutarch, Lycurgus VI: C. What is the Rhetra?", CQ 38.3/4 (1944) 115-126.

Wallace 2012: R. Wallace, "Charismatic Leaders", en K. A. Raaflaub, H. van Wees, A Companion to Archaic Greece (New York 2012) 411-426.

Whitby 2002: M. Whitby (ed.), Sparta (New York - London 2002).

Zweig 1993: B. Zweig, "The Only Women Who Give Birth to Men: A Gynocentric, Cross-Cultural View of Women in Ancient Sparta", en M. DeForest (ed.), Woman's Power, Man's Game: Essays on Classical Antiquity in Honor of Joy K. King (Illinois 1993) 32-53. 